\title{
USING ARTIFICIAL INTELLIGENCE TO DETERMINE THE TYPE OF ROTARY MACHINE FAULT
}

\author{
Daniel Zuth, Tomáš Marada \\ Brno University of Technology \\ Faculty of Mechanical Engineering \\ Institute of Automation and Computer Science \\ Technická 2896/2, 616 69, Brno \\ Czech Republic \\ zuth@fme.vutbr.cz, ORCID: 0000-0001-9543-8811 \\ marada@fme.vutbr.cz, ORCID: 0000-0003-2141-4977
}

\begin{abstract}
The article deals with the possibility of using machine learning in vibrodiagnostics to determine the type of fault of rotating machine. The data source is real measured data from the vibrodiagnostic model. This model allows simulation of some types of faults. The data is then processed and reduced for the use of the Matlab Classification learner app, which creates a model for recognizing faults. The model is ultimately tested on new samples of data. The aim of the article is to verify the ability to recognize similarly rotary machine faults from real measurements in the time domain.
\end{abstract}

Keywords: Vibrodiagnostics, Neuron Network, Classification Learner, Machine Learning, Matlab, Industry 4.0, Classification Method, Static Unbalance, Dynamic Unbalance

\section{Introduction}

The arrival of Industry 4.0 requires the use of new methods in classical engineering fields such as autonomous and/or predictive elements in diagnostics. Moving of most human activities to the machine in diagnostics can reduce economic losses through timely intervention.

The article will deal with the use of artificial intelligence (see, e.g. $[3,4,5]$ ) in the area of vibrodiagnostics, namely the determination of the fault of the rotary machine from the measured sample in the time domain. The data are obtained from two MEMS (MicroElectroMechanical Systems) accelerometers ADXL335 [9] located on the bearing stands (positions 1 and 2) of Fig. 2. They are analog accelerometers, the output signal is processed by the NI USB-6009 [10] DAQ card with the $2 \mathrm{kHz}$ sampling frequency and 1,000 samples settings. The sensor is mount with screws to a printed plastic part that is bolted to the mounting magnet (Fig. 1). The experiment is divided into a one-point measurement with one sensor and a two-point measurement from two sensors simultaneously. Basic faults (static and dynamic unbalance) are simulated by two levels and compared to a faultless state.

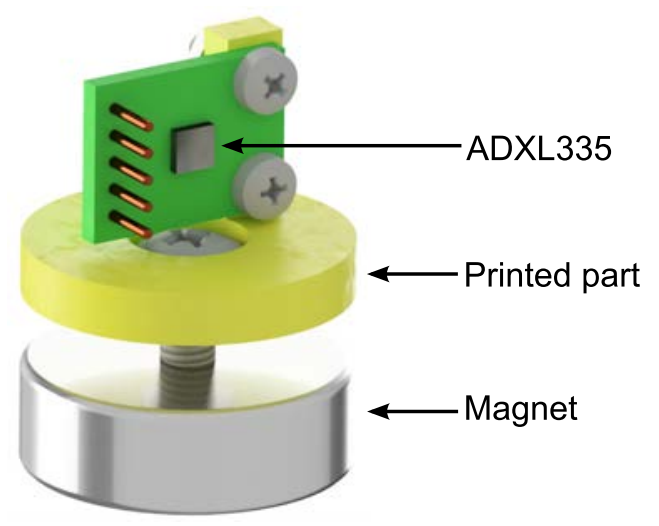

Figure 1: Sensor with mounting magnet 
Basic features of the sensor [9] :

- Measuring range: $\pm 3 \mathrm{~g}$

- Bandwidths for X and Y axes: $0.5 \mathrm{~Hz}$ to $1,600 \mathrm{~Hz}$

- Bandwidths for $\mathrm{Z}$ axis: $0.5 \mathrm{~Hz}$ to $550 \mathrm{~Hz}$

Basic features of DAQ card [10] :

- Resolution ADC: 14 bit

- Sample frequency: $48 \mathrm{kSps}$

- Channels: 8 AI, 2 AO, 13 DIO

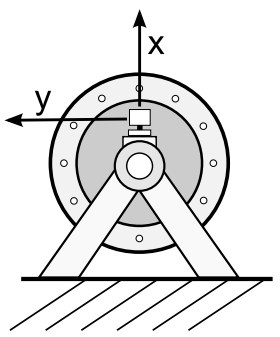

a)

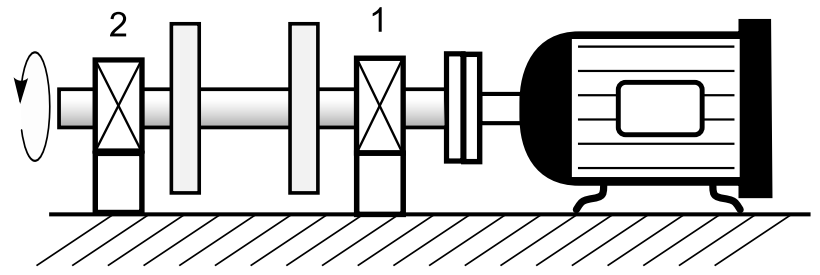

b)

Figure 2: Model for fault simulation a) Left side with shown the axes b) Front side with shown the positions

\section{Faults Simulation}

The unbalance faults are possible to simulate on the used model (schematically illustrated in Fig. 2). The unbalance may be static and dynamic, which differ from each other mainly by shifting the phase of the signal between bearing stands in the same axis [1], [2], [6]. The difference in the simulation of dynamic and static unbalance is in the placement of weights on rotary discs. The location of the weight is shown in Fig. 3. Unbalance weight for level 1 is 2.5 grams and weight for level 2 is 3.7 grams. The rotational speed was set at $1,190 \mathrm{rpm}$ for all measurements, the first harmonic component was calculated as $19.83 \mathrm{~Hz}$.

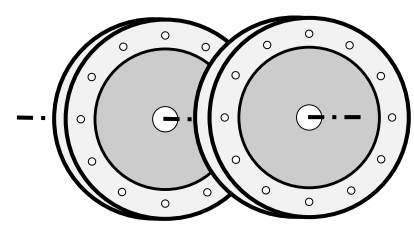

a)

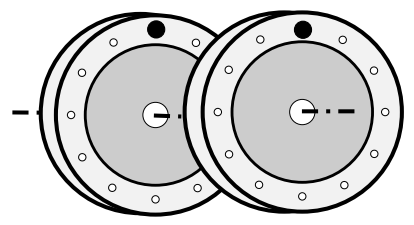

b)

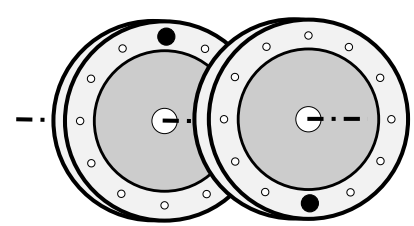

c)

Figure 3: Scheme of weights and positions a) Faultless state b) Static unbalance c) Dynamic unbalance

\section{Distribution of Faults in Classification Classes}

Five different measurements were recorded, namely faultless state, static unbalance with lighter and heavier weights and dynamic unbalance with lighter and heavier weights. The following five classes were created and named: 
Class 1 "OK" - Both weights are removed. This is a faultless state (Fig. 3a)

Class 2 "ST1" - Static unbalance level 1 - Weights of 2.5 grams are placed on both discs (Fig. 3b)

Class 3 "DYN1" - Dynamic unbalance level 1 - Weights of 2.5 grams are placed on both discs (Fig. 3c)

Class 4 "ST2" - Static unbalance level 2 - Weights of 3.7 grams are placed on both discs (Fig. 3b)

Class 5 "DYN2" - Dynamic unbalance level 2 - Weights of 3.7 grams are placed on both discs (Fig. 3c) The waveform signals of the individual faults in the individual axes are shown in Fig. 4.
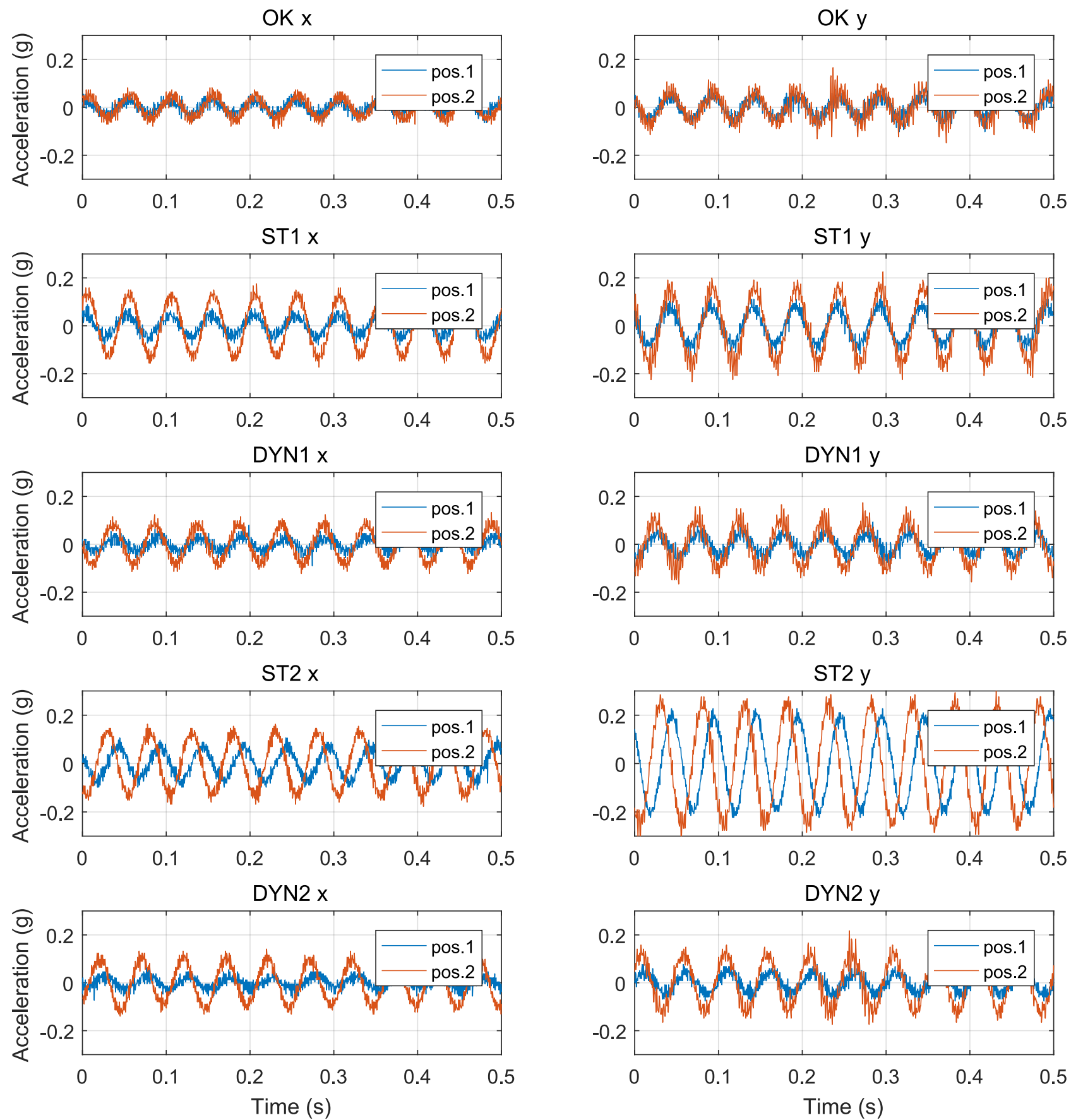

Figure 4: Measured data of all states (one random sample of measurements) 


\section{Data Processing}

The classification of individual faults is evaluated from a measured sample of data in the time domain (not the frequency domain) as measured from a DAQ card with a sampling frequency $2 \mathrm{kSps}$ and 1,000 samples. This signal was processed by the following functions.

- RMS function - The RMS value is commonly used in vibrodiagnostics to determine machine condition [1], [2], [6], [7], [8]. The Matlab rms () function is used.

- STD function - The standard deviation. The Matlab std() function was used.

- PCA function - The principal component analysis of raw data. The Matlab pca() function was used.

- Phase shift - The estimate delay(s) between the signals. The Matlab finddelay() function was used (marked as FDx and FDy).

a)

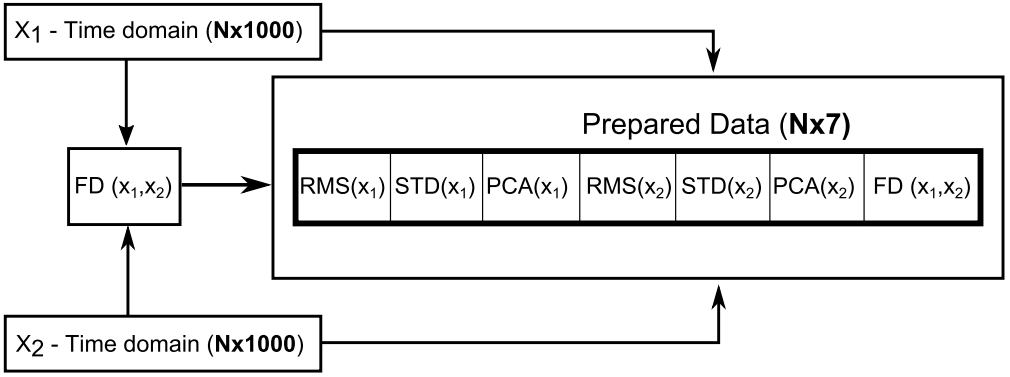

Prepared Data $\mathbf{( N \times 1 4 )}$

b)

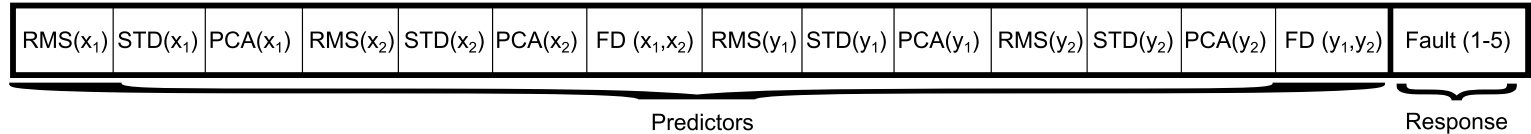

Prepared Data (Nx6)

c)

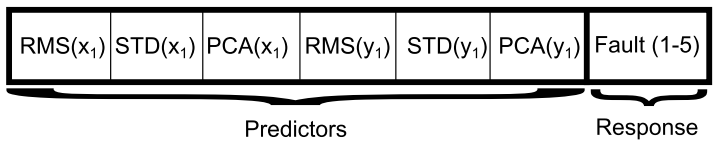

Figure 5: a) Processing of data for one axis from two positions

b) The resulting dataset from both positions for both axes - P2 c) The dataset from one sensor - P1

\section{Classifier Selection}

The Classification Learner App can test the dataset for different classification methods and choose the most successful one for generating the classification model. The experiment was divided into two variants: measuring only one position with one sensor (P1) and measuring both positions simultaneously (P2). This division was used for the success of classification comparison if we have information from only one sensor. The predictors for the P1 variant are RMSx, STDx, PCAx, RMSy, STDy, PCAy (Figure 5c), thus the data was reduced from Nx1000 to Nx6. The predictors for the P2 variant are RMS, STD, PCA - for both position and axis and FDy and FDx (Fig. 5b), thus the data was reduced from Nx1000 to Nx14. The successes rates of both variants are compared in Tab. 1.

The best result for the P1 variant was reached with Linear SVM (Support Vector Machines) method, the success rate reached $90.2 \%$ (Tab. 1). This model was chosen for the resulting classifier. Most models for the P2 variant was reached $100 \%$ success (Tab. 1). The same model as the P1 variant (Linear SVM) was chosen for the resulting classifier. 


\section{Verification of the Classification Model}

The new datasets for testing the new classification model contain 500 new measurements for each fault. The success of the fault classification is shown in Fig. 6. The Trained Classifier for variant P1 reached the success rate $91.08 \%$ (2,277 true predicted, 223 false predicted). The Trained Classifier for variant P2 reached the success rate $100 \%$ (2,500 true predicted, 0 false predicted).

Table 1: The comparison of the classification methods from Classification Learner App

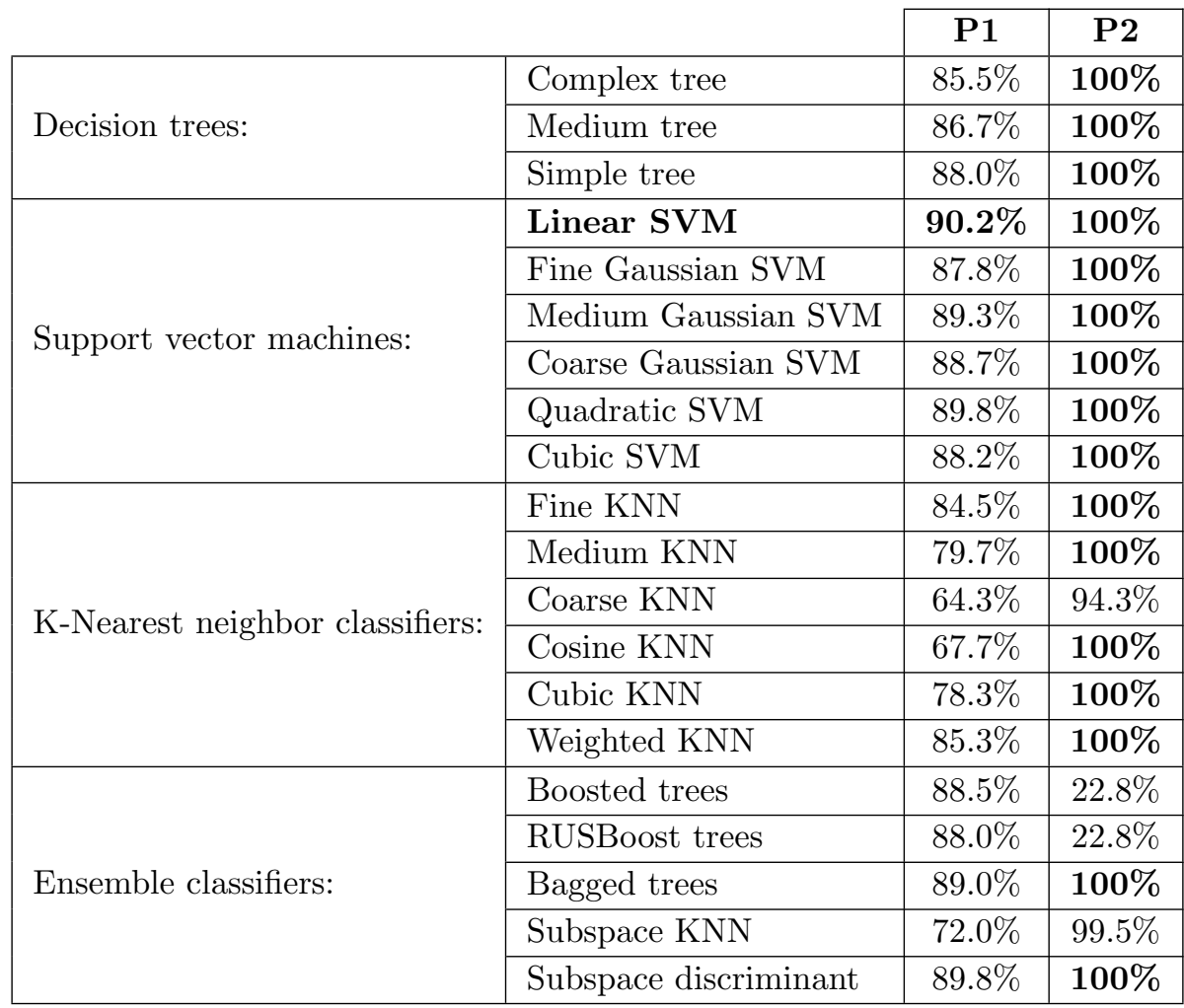

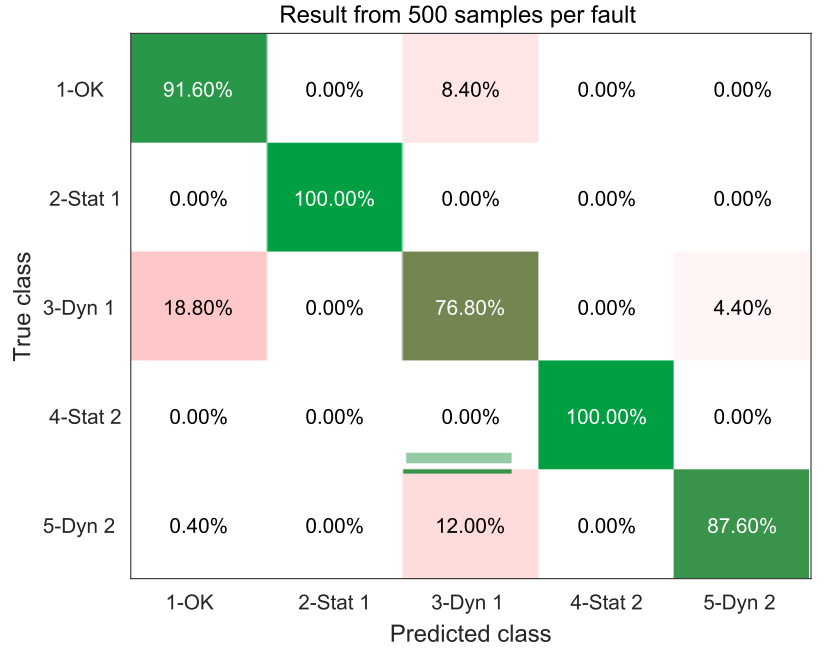

a)

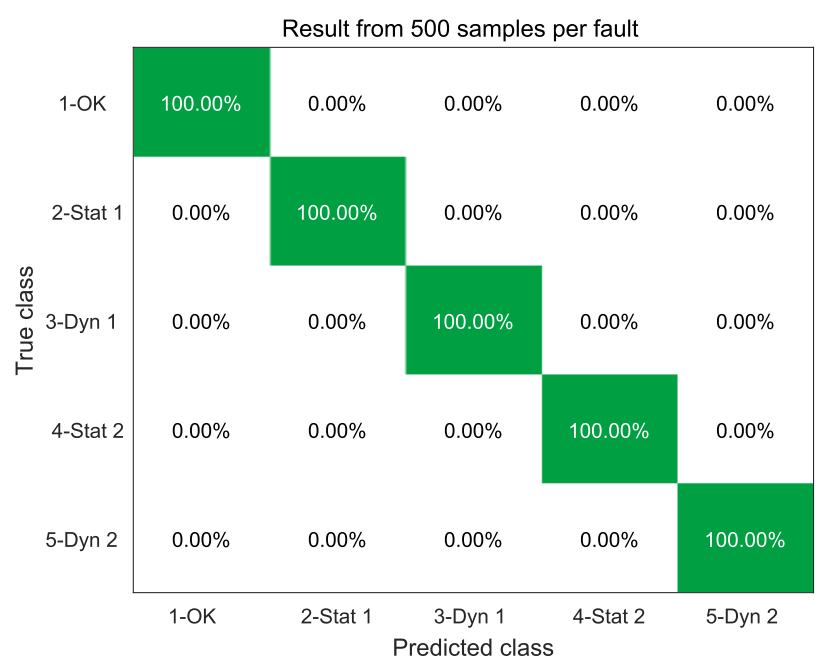

b)

Figure 6: The results of the generated classification model

a) Variant P1 b) Variant P2 


\section{Conclusion}

The article verifies the ability of the rotary machine faults classification from the measurement data in the time domain. Five states were compared, namely a faultless state and static and dynamic unbalance with two different weights. Furthermore, the classification success rate was compared, assuming data capture by one sensor at one position (P1) and assuming two positions and two sensors simultaneously (P2).

The results were summarized in Fig. 6, which compares variants P1 and P2. Future experiments will include disturbing effects of measurement such as changing the rotary speed or adding another type of faults (for example, "Loose base"). Experiments that do not contain disturbing effects reached high classification success. The best result for the P1 variant was reached with Linear SVM model, the success rate reached $90.2 \%$ (Tab. 1). Most models for the P2 variant reached 100\% success (Tab. 1).

Acknowledgment: This research was supported by the grant No. FSI-S-17-4785.

\section{References}

[1] Broch, J.T.: Mechanical Vibration and Shock Measurements, Naerum, Denmark (1984). ISBN 8787355361

[2] Blata, J., Juraszek, J.: Metody technické diagnostiky, teorie a praxe [in Czech]. REPRONIS, s.r.o.: Ostrava (2013). ISBN 978-80-248-2997-5

[3] Castellani, M., Lalchandani, R.: An experimental study on competitive coevolution of MLP classifiers. Mendel 23(1), 41-48 (2017)

[4] Ren, Y., Suganthan, P.N., Srikanth, N., Amaratunga, G.: Random vector functional link neural network for short-term wind power ramp forecasting. Mendel 21(1), 77-87 (2015)

[5] Zuth, D.: Using HIL simulation and genetic algorithms for controller tuning. Mendel 22(1), 25-30 (2016)

[6] Zuth, D., Vdoleček, F.: Měření vibrací ve vibrodiagnostice [in Czech]. Automa 16(1), 32-36 (2010)

[7] Sedenka, D., Blata, J., Kasiar, L., Heisig, L., Zarsky, V.: Deployment of technical diagnostics during commissioning of small pumped storage hydropower plant. International Multidisciplinary Scientific GeoConference Surveying Geology and Mining Ecology Management, SGEM, 17(42), 175-182 (2017)

[8] Vdoleček, F.: Terminologie v oboru nejistot merení [in Czech]. Akustika, 16(1), 40-42 (2012)

[9] ADXL335 Datasheet and Product Info - Analog Devices http://www.analog.com/en/products/sensorsmems/accelerometers/adxl335.html. [Online; accessed 20-Apr-2018]

[10] USB-6009 - National Instruments http://www.ni.com/cs-cz/support/model.usb-6009.html. [Online; accessed 20-Apr-2018]

[11] Statistics and Machine Learning Toolbox - MathWorks - Makers of MATLAB and Simulink https://ch.mathworks.com/help/stats/. [Online; accessed 20-Apr-2017]

[12] Train models to classify data using supervised machine learning - MathWorks - Makers of MATLAB and Simulink https://www.mathworks.com/help/stats/classificationlearner-app.html. [Online; accessed 20-Apr-2017] 